\title{
Multispectral infrared detection using plasmon-assisted cavities
}

R. V. Shenoi, J. Rosenberg, T. E Vandervelde, O. J. Painter, S. Krishna

R. V. Shenoi, J. Rosenberg, T. E Vandervelde, O. J. Painter, S. Krishna, "Multispectral infrared detection using plasmon-assisted cavities," Proc. SPIE 7298, Infrared Technology and Applications XXXV, 729808 (7 May 2009); doi: $10.1117 / 12.818982$

SPIE Event: SPIE Defense, Security, and Sensing, 2009, Orlando, Florida, United States 


\title{
Multispectral Infrared Detection Using Plasmon-assisted Cavities.
}

\author{
R. V. Shenoi ${ }^{a}$, J. Rosenberg ${ }^{b}$, T. E. Vandervelde ${ }^{c}$, O. J. Painter ${ }^{b}$, and S. Krishna ${ }^{a}$ \\ ${ }^{a}$ Center for High Technology Materials, ECE Department, University of New Mexico, 1313 Goddard \\ St. SE, Albuquerque, New Mexico 87106; \\ ${ }^{b}$ Thomas J. Watson, Sr., Laboratory of Applied Physics, California Institute of Technology, Pasadena, \\ CA 91125; \\ ${ }^{c}$ Electrical and Computer Engineering Department, Tufts University, 161 College Ave, Medford, MA \\ 02155 .
}

\begin{abstract}
We report the demonstration of multi-spectral quantum dots-in-a-well infrared photo-detectors through the coupling of incident light to resonant modes of surface plasmons. The integration of a surface plasmon assisted cavity with the detector results in shifting the peak wavelength of absorption of the detector to that of the resonant wavelength of the cavity. The cavity consists of a square lattice structure with square holes in it. A wavelength tuning of 8.5 to $9 \mu \mathrm{m}$ was observed, by changing the pitch of the fabricated pattern forming the cavity. Polarization sensitive detectors can be fabricated by breaking the symmetry of the lattice. This is achieved by stretching the lattice constants along the $\mathrm{x}$ and $\mathrm{y}$ directions. A DWELL detector with resonant frequency at $6.8 \mu \mathrm{m}$ where the response of the $0^{\circ}$ polarization is twice as strong as the $90^{\circ}$ polarization is reported. This technique, in principle, is detector agnostic and shows promise in fabrication of multi-spectral focal plane arrays (FPA).
\end{abstract}

Keywords: Quantum Dots, Infrared detector, Dots-in-a-Well Detector, Multi-spectral detection, Plasmons

\section{INTRODUCTION}

Infrared detectors are widely used in the mid-wave infrared (MWIR, 3-5 $\mu \mathrm{m}$ ) and long-wave infrared (LWIR, 8-12 $\mu \mathrm{m}$ ) regions, for applications in thermography, imaging, missile defense, and medical diagnostics. ${ }^{1,2}$ The major technologies currently used in this field are mercury cadmium telluride (MCT), indium antimonide ( $\mathrm{InSb}$ ) and quantum well infrared photodetectors (QWIP). Quantum dot infrared photodetectors (QDIP) and strained layer superlattice (SLS) are considered to be the emerging technologies. Higher temperature operation, large area arrays and multispectral operation are considered to be the requirements for the third generation infrared detectors. QDIPs leverage the mature growth and processing technologies of III-V semiconductors, which makes the fabrication of large sized arrays possible. Improved designs to enhance the quantum efficiency and lower the dark current have resulted in higher operating temperatures. QDIPs have been reported to operate at temperatures as high as $200 \mathrm{~K}$ in the mid-wave infrared region (MWIR) and $140 \mathrm{~K}$ in the longwave infrared (MWIR) regions. ${ }^{3,4}$ Several techniques have been proposed to fabricate multi-spectral detection using these technologies. The use of two back-to-back biased diodes, each responding to different wavelengths is one of the common approaches. These devices use two contacts per pixel to fabricate two-color detectors. Changing the detection wavelength by changing the bias applied on the detector is another technique. This approach is especially attractive, as it eliminates the complex processing steps required to fabricate multiple contacts per pixel. A variant of the QDIP known as the quantum dots-in-a-well (DWELL) infrared photodetector, where the quantum dots are embedded in a quantum well shows a bias tunable response. ${ }^{5}$ This is achieved by designing the width of the quantum well above and below the well asymmetrically, resulting in different wavelength responses in the positive and negative bias. The detection of multiple wavelengths, still remains a challenge.

The efforts to develop multispectral detectors have been mainly dependant on the integration of resonant cavities or gratings with detectors. Fabry-Perot (FP) cavities are the most commonly used ones. However fabrication of these cavities with low full width half maxima (FWHM) and integration of these cavities with the active region still make the fabrication

Corresponding author: R. V. Shenoi

E-mail: rshenoi@ece.unm.edu

Infrared Technology and Applications XXXV, edited by Bjørn F. Andresen, Gabor F. Fulop, Paul R. Norton Proc. of SPIE Vol. 7298, 729808 - (C) 2009 SPIE · CCC code: 0277-786X/09/\$18 · doi: 10.1117/12.818982 
of multi-spectral detectors difficult. ${ }^{6}$ The active region is sandwiched between the FP cavity during the growth of the detector active region. This also leads to additional processing steps to fabricate different bands of absorption in the same active region. Another approach is the use of diffractive gratings. This has resulted in improving the performance of QWIPs in the LWIR region. The gratings etched into the active region, couple the incident light into the higher order modes enabling higher absorption. ${ }^{7,8}$ The use of photonic crystal based cavities have enhanced the absorption characteristics of DWELL detectors, without degrading the noise performance. ${ }^{9}$ Tuning of the peak wavelength of the detector, however remains an attractive option for fabricating multi-spectral detectors. The use of a common active region and use of post-growth techniques to achieve multi-spectral detection can help to bring down the cost of a focal plane array (FPA) drastically.

A multi-spectral DWELL infrared photodetector, where the detection of multiple wavelengths is achieved through the coupling of incident light to surface plasmons that exist at a metal-semiconductor layer is described in this paper. The coupling is made possible by the fabrication of a two-dimensional patterned metal layer on the detector aperture, which results in the excitation of surface plasmons. The resonant wavelength of the detector pixel can be changed by varying the pitch of the fabricated pattern. This provides an easy way to fabricate multi-spectral detectors using the same active region. Additional information like polarization, can be can be encoded in the response of the detector by varying the pitch along the orthogonal directions. The fabrication process is simple, detector agnostic and can be easily transformed into a FPA.

\section{DESIGN AND GROWTH}

The detectors active regions consisted of InAs dots embedded in quantum wells. Two designs were considered, where the dots were embedded in a single or double well. The single well design consisted of 15 stacks of InAs dots embedded in $\mathrm{In}_{0.15} \mathrm{Ga}_{0.85}$ As wells with GaAs as the barrier. ${ }^{1}$ The alternate design consists of InAs dots in $\operatorname{In}_{0.15} \mathrm{Ga}_{0.85} \mathrm{As} / \mathrm{GaAs}$ wells with $\mathrm{Al}_{0.10} \mathrm{Ga}_{0.90}$ As as the barrier. ${ }^{10}$ This design allows the growth of 30 stacks of active region due to the reduced strain per layer. The contact regions consisted of n-GaAs doped to $2 \times 10^{18} / \mathrm{cm}^{-3}$. A $2 \mu \mathrm{m}$ thick $\mathrm{Al}_{0.7} \mathrm{Ga}_{0.3}$ As below the active region as a cladding layer for the confinement of the mode in the active region. The detectors samples were then grown by using a VG-80 solid source molecular beam epitaxy (MBE) reactor.
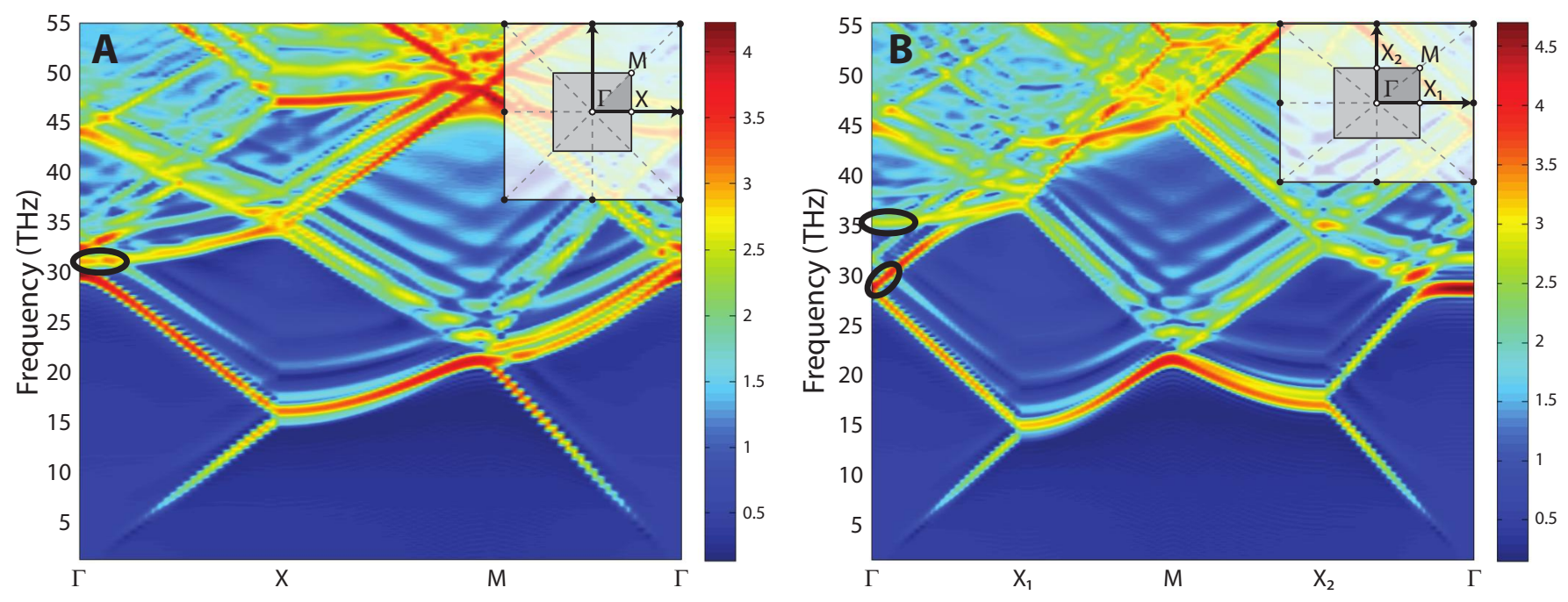

Figure 1. A) Bandstructure of a square lattice with square holes. The flat-band regions near the $\Gamma$ point are marked. B) Splitting of bands in a square lattice structure by stretching and compressing lattice constants in orthogonal directions. The split in frequency of modes is indicated.

Finite difference time domain (FDTD) simulations were performed to model the effect of the plasmon metal layer on the response of the detector. The metal layer consisted of a thin layer of silver about $150 \mathrm{~nm}$ thick, with square holes etched in a square lattice. The simulations showed a hole width $/ a$ ratio of 0.567 and a a/ $\lambda$ of 0.31 . The attempt was to couple to the band-edge modes of this structure at the $\Gamma$ point, that correspond to the coupling of normal incident light. 
Due to the symmetry, the square lattice has two degenerate modes at the $\Gamma$ point to which the incident light couples. This symmetry can be broken by changing the lattice constant of the pattern along the two orthogonal directions. This results in splitting of the modes in frequency. These modes show a highly polarization dependant coupling, which can be utilized to fabricate polarization sensitive detectors, which can used in applications like target detection. ${ }^{11} \mathrm{~A}$ band structure of the square lattice, showing the bands at $\Gamma$ point and the resulting frequency split as a result of breaking the symmetry, are shown in Fig. 1 A) and B).
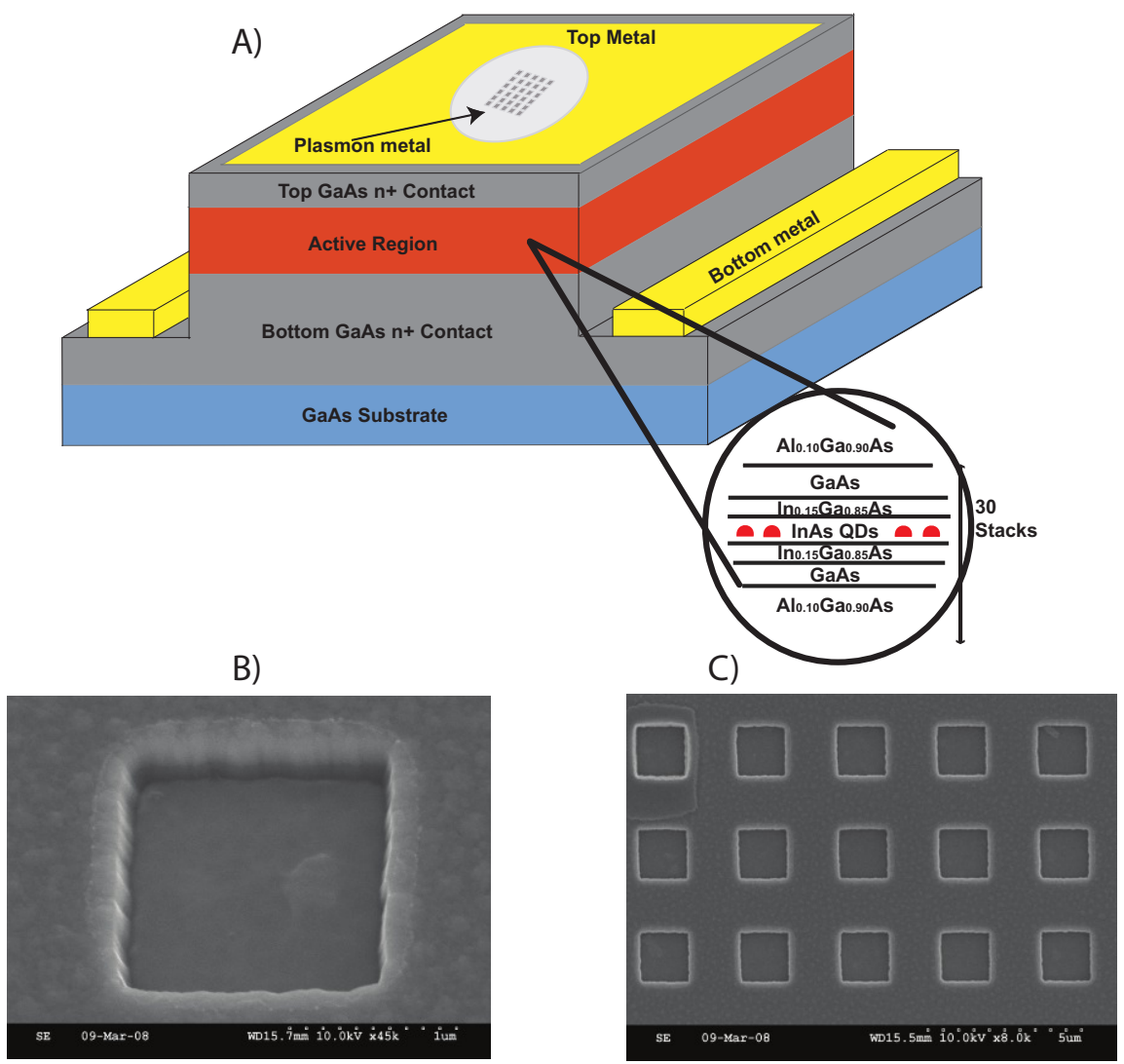

Figure 2. A) Schematic of the fabricated detector. The top and bottom metal layer and the plasmon metal layer covering the aperture is shown. B), C). Scanning electrom microscope (SEM) images of the fabricated photonic crystal.

The detector pixels were then fabricated using photolithography and dry etch. The detector side-walls were then passivated using a $S i N_{x}$ passivation layer and $\mathrm{Ge} / \mathrm{Au} / \mathrm{Ni} / \mathrm{Au}$ was deposited for contacting the n-GaAs using an e-beam evaporator, creating apertures ranging from 25 to $300 \mu \mathrm{m}$. The samples were then annealed at $380{ }^{\circ} \mathrm{C}$ to obtain an ohmic contact. Following the annealing, a thin layer of Ti/Ag was deposited, covering the aperture of the detector. This metal layer serves to excite the plasmons at the metal-semiconductor interface. Square lattices with square holes were fabricated on the plasmon metal layer of these samples (set A) using e-beam lithography and dry etching. The lattice constants were varied while keeping the width (w) to pitch (a) ratios constant. A different set of samples (set B) were also fabricated where the lattice constants were stretched in one direction and compressed in a different direction. A schematic of the detector and and SEM image of the patterned top layer are shown in Fig. 2 (A) and (B) respectively.

\section{CHARACTERIZATION}

The spectral response of the devices, as a function of the applied bias voltage were characterized using a Nicolet 870 Fourier Transform Infrared Spectrometer. Spectral response of the detectors with the patterned plasmon metal layer was 
measured and compared with a control sample. The control sample consists of a detector from the same sample as the patterned devices, but without the plasmon metal layer. This allows the observation and characterization of the effects of the patterned metal layer on the detector response, while keeping the variations in growth and processing to a minimum.

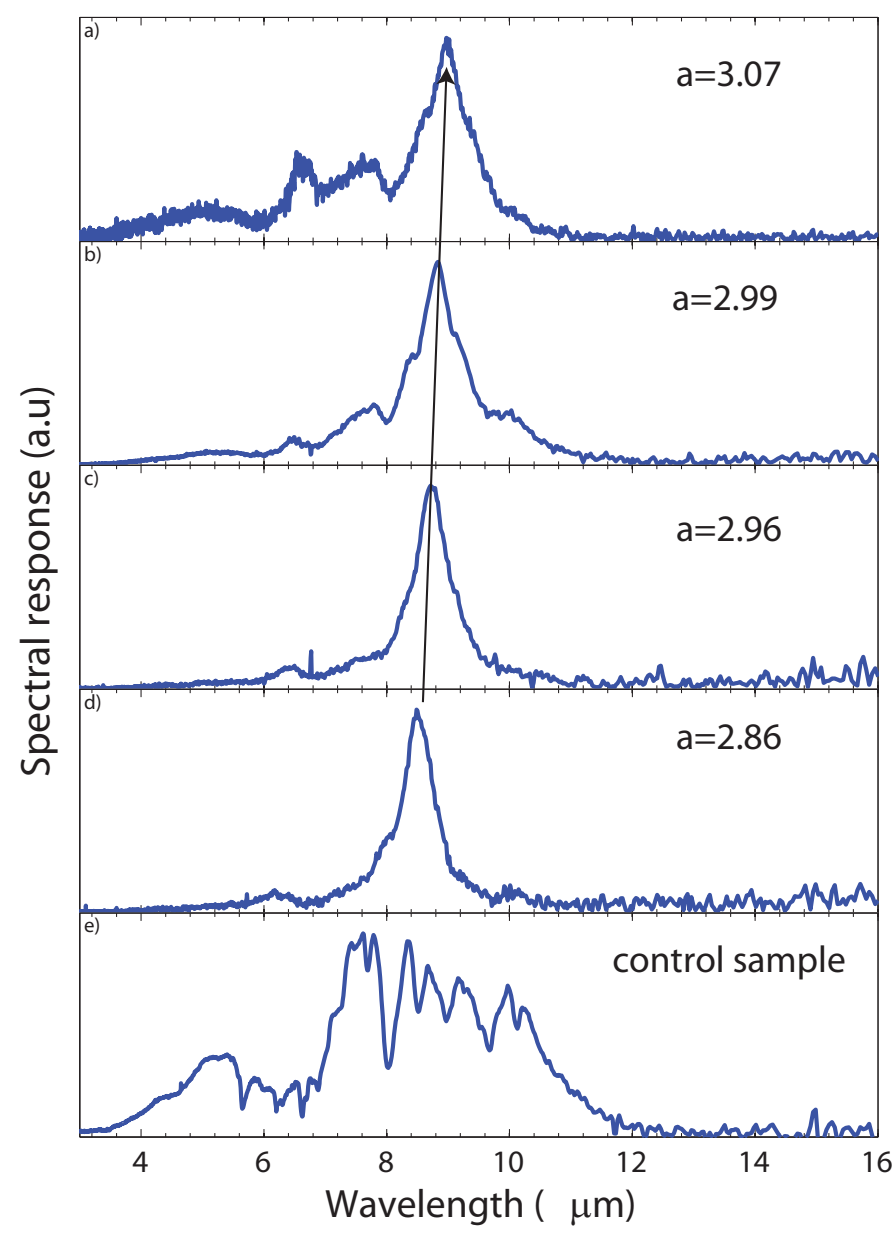

Figure 3. Spectral response measured at $4.0 \mathrm{~V}$ and $30 \mathrm{~K}$ for the control sample and samples with a patterned plasmon layer, as a function of the pitch of the pattern. The peak wavelength of response shifts with the introduction of the nanostructured metal layer and increases as the pitch increases. A wavelength tuning from 8.5 to $9 \mu \mathrm{m}$ is observed.

Spectral response of the patterned devices and that of control sample, for positive and negative bias voltages are shown in Fig. 3 and Fig. 4 respectively. The response was measured at a temperature of $30 \mathrm{~K}$. The control samples were measured for bias voltages of $4.0 \mathrm{~V}$ and $-4.2 \mathrm{~V}$ respectively. For the positive bias, they show a broad peak ranging from 7 to $10.4 \mu \mathrm{m}$ and for the negative bias, a LWIR peak of $10.3 \mu \mathrm{m}$ with a full width half maxima of $1.4 \mu \mathrm{m}$. Response can also be observed in the negative bias from 4.9 to $7.8 \mu \mathrm{m}$ with a lower intensity. At low bias voltages, the MWIR peak, due to transitions from states in the quantum dot to quasi-bound states is dominant. As the bias voltage increases, the intensity of the LWIR peak, due to transitions from energy levels in the quantum dot to states in the quantum well, increases. ${ }^{1}$ The well widths above and below the quantum dot are asymmetric in a DWELL structure, leading to the bias dependant spectral response as a result of the quantum confined Stark effect (QCSE). ${ }^{12}$

The response of the patterned devices for a positive bias shows a single peak, with the peak wavelength varying from 8.5 to $9 \mu \mathrm{m}$ with the change in the lattice constant of the pattern. With increasing lattice constant, the peak wavelength 


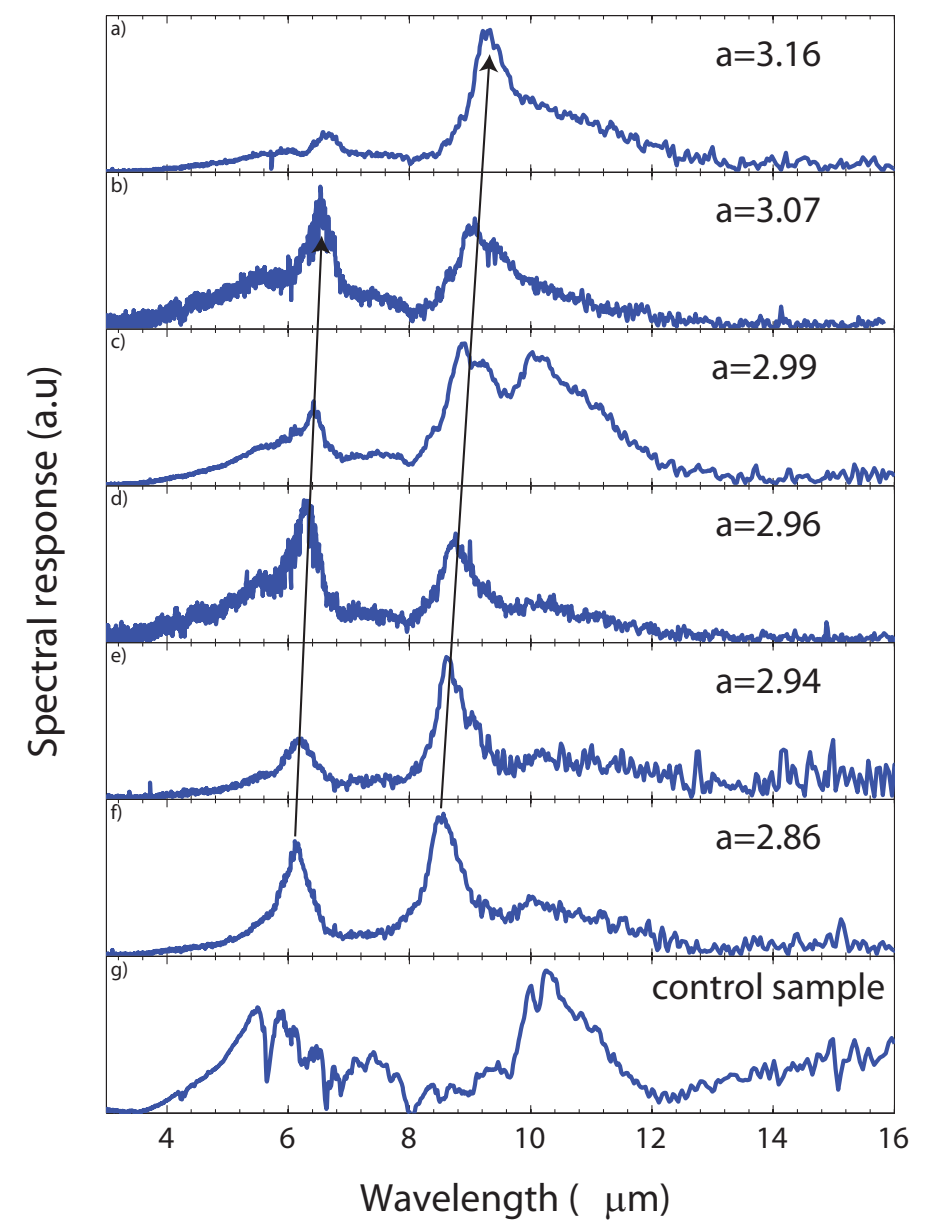

Figure 4. Spectral response measured at $-4.2 \mathrm{~V}$ and $30 \mathrm{~K}$ as a function of the pitch of the pattern. The fundamental mode of the structure is observed to vary from $8.5 \mu \mathrm{m}$ to $9.3 \mu \mathrm{m}$ with the change in the pitch of the pattern. A higher order mode can be observed at lower wavelengths, with the peak varying from $6.1 \mu \mathrm{m}$ to $6.6 \mu \mathrm{m}$.

increases. The peaks show a lower FWHM of $900 \mathrm{~nm}$, indicating a quality factor (Q) of 10. For the negative bias response at $-4.2 \mathrm{~V}$, the patterned samples show two peaks, with the LWIR peak varying from $8.5 \mu \mathrm{m}$ to $9.3 \mu \mathrm{m}$ and a second peak varying from $6.1 \mu \mathrm{m}$ to $6.6 \mu \mathrm{m}$. The presence of a second peak at a lower wavelength could be due to a higher order mode of the structure. The FWHM for these peaks is around 800-900 nm, which is lower than that of the control sample. These measurements indicate that, with the integration of a plasmon layer to the DWELL detector it is possible to shift the peak wavelength of absorption of the detector and that it can be tuned by varying the pitch of the pattern. This provides a cost-effective method for development of multi-spectral detectors, as it utilizes the same active region and avoids the complicated processing needed to fabricate multiple indium bumps per pixel.

In order to ascertain the polarization dependence of the spectral response, the samples in set B were measured with a wiregrid polarizer. A plot of the spectral response of these samples at a temperature of $77 \mathrm{~K}$ and a bias voltage of 5 $\mathrm{V}$ is shown in Fig. 5. The unpolarized response shows a resonant peak at $6.8 \mu \mathrm{m}$. This peak is visible only at an input polarization of $0^{\circ}$, indicating the strongly polarized nature of the resonant peak. The weak response from the other split-off mode of the pattern can be observed at $90^{\circ}$ polarization at around $8 \mu \mathrm{m}$. The response at $6.8 \mu \mathrm{m}$ for the $0^{\circ}$ polarized light is almost twice as strong as the $90^{\circ}$ polarized light. 


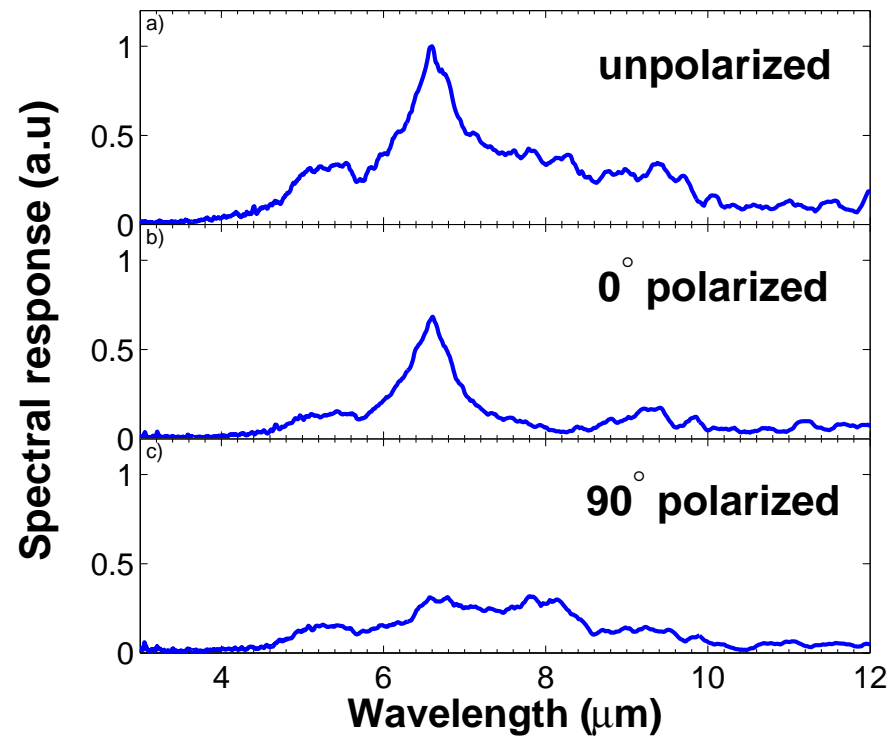

Figure 5. Spectral response from a sample with stretched and compressed lattice constants at $5 \mathrm{~V}$ bias and $77 \mathrm{~K}$ as a function of the polarization angle.

\section{CONCLUSION AND FUTURE WORK}

In conclusion, we report the fabrication of multi-spectral polarization sensitive DWELL detectors by the coupling of incident light into the resonances of surface plasmons existing at a metal-semiconductor interface. The coupling is achieved by patterning a thin metal layer deposited at the aperture of the detector with square holes in a square lattice structure. The peak response of the detector is tuned from $8.5 \mu \mathrm{m}$ to $9 \mu \mathrm{m}$ by changing the pitch of the pattern. The samples also show a higher order mode of the pattern in the negative bias. This opens up the possibility of multi-spectral detection in the same pixel of the detector through optimized design of the pattern. Polarization sensitive devices are demonstrated by breaking the symmetry of the square lattice structure, which separates the degenerate modes of the pattern in frequency. A polarization sensitive detector was demonstrated at $6.8 \mu \mathrm{m}$ with the resonance dominated by the $0^{\circ}$ polarization. The detector agnostic nature and simplicity of this technique can help to fabricate multi-spectral FPAs cheaply. The optimization of the patterns, however is far from complete and would be subject to future work. The use of defect-mode cavities and their optimization would also be pursued.

\section{ACKNOWLEDGMENTS}

This work was funded by AFOSR.

\section{REFERENCES}

[1] S.Krishna, "Quantum dots-in-a-well infrared photodetector," J. Phys. D 38, 2142 (2005).

[2] Rehm, R., Walther, M., Schmitz, J., Fleiner, J., Ziegler, J., Cabanski, W., and Breiter, R., "Dual-colour thermal imaging with InAs/GaSb superlattices in mid-wavelength infrared spectral range," Electronic Letters 42, 2124-2127 (2006).

[3] Zhang, W., Lim, H., Taguchi, M., Tsao, S., Movaghar, B., and Razeghi, M., "High-detectivity InAs quantum-dot infrared photodetectors grown on InP by metalorganic chemical vapor deposition," Appl. Phys. Lett. 86, 191103 (2005).

[4] Shenoi, R. V., Hou, J., Vandervelde, T. E., Shao, J., and Krishna, S., "High operation temperature of a low strain quantum dots-in-a-double-well infrared photodetector," To be published . 
[5] Vandervelde, T., Lenz, M., Varley, E., Barve, A., Shao, J., Shenoi, R., Ramirez, D., Jan, W., Sharma, Y., and Krishna, S., "Quantum dots-in-a-well focal plane arrays," Selected Topics in Quantum Electronics, IEEE Journal of 14, 11501161 (July-Aug. 2008).

[6] Attaluri, R. S., Shao, J., Posani, K. T., Lee, S. J., Brown, J. S., Stintz, A., and Krishna, S., "Resonant cavity enhanced InAs/In ${ }_{0.15} \mathrm{Ga}_{0.85}$ As dots-in-a-well quantum dot infrared photodetector," J. Vac. Sci. Tech B. 25, 1186 (2007).

[7] Schimert, T. R., Barnes, S. L., Brouns, A. J., Case, F. C., Mitra, P., and Claiborne, L. T., "Enhanced quantum well infrared photodetector with novel multiple quantum well grating structure," Applied Physics Letters 68(20), 28462848 (1996).

[8] Schartner, S., Golka, S., Pflugl, C., Schrenk, W., Andrews, A. M., Roch, T., , and Strasser, G., "Band structure mapping of photonic crystal intersubband detectors," Appl. Phys. Lett. 89, 151107 (2006).

[9] Posani, K. T., Tripathi, V., Annamalai, S., Weisse-Bernstein, N. R., Krishna, S., Perahia, R., Crisafulli, O., and Painter, O. J., "Nanoscale quantum dot infrared sensors with photonic crystal cavity," Appl. Phys. Lett. 88, 151104 (2006).

[10] Shenoi, R. V., Attaluri, R. S., Siroya, A., Shao, J., Sharma, Y. D., Stintz, A., Vandervelde, T. E., and Krishna, S., "Low-strain InAs/InGaAs/GaAs quantum dots-in-a-well infrared photodetector," J. Vac. Sci. Tech B. 26, 1136-1139 (2008).

[11] Tyo, J. S., Goldstein, D. L., Chenault, D. B., and Shaw, J. A., "Review of passive imaging polarimetry for remote sensing applications," Appl. Optics 45, 5453-5469 (2006).

[12] Krishna, S., Raghavan, S., von Winckel, G., Stintz, A., Ariyawansa, G., Matsik, S. G., and Perera, A. G. U., "Threecolor $\left(\lambda_{p 1} \approx 3.8 \mu \mathrm{m}, \lambda_{p 2} \approx 8.5 \mu \mathrm{m}\right.$, and $\left.\lambda_{p 3} \approx 23.2 \mu \mathrm{m}\right)$ InAs/InGaAs quantum-dots-in-a-well detector," Appl. Phys. Lett. 83, 2746 (2003). 